https://doi.org/10.1007/s00337-019-0511-0

Online publiziert: 1. April 2019

(c) Der/die Autor(en) 2019
Manuelle Medizin 2019 ·57:111-115

Titus Schultz

Allmannsweier, Deutschland

\section{Die manuelle Behandlung des lliosakralgelenks nach Operation an Lendenwirbelsäule und Hüftgelenk}

\section{Fragestellung}

Bei ca. $50 \%$ der Patienten, die sich nach Implantation einer Hüfttotalendoprothese (HTEP) oder nach Operation der Lendenwirbelsäule (LWS) einer Rehabilitation unterziehen, besteht eine Beschwerdekonstellation mit

- schmerzhafter Gangstörung,

- Leistenschmerzen nach HTEPImplantation,

- sensiblem Pseudo-L5/S1-Syndrom,

- Bewegungseinschränkung des betroffenen Hüftgelenks,

- Hilfebedarf beim Strümpfeanziehen sowie

- Schwierigkeiten beim Treppensteigen (alternierend nicht möglich).

Bei der manuellen Untersuchung findet sich nahezu regelmäßig eine Dysfunktion des Iliosakralgelenks (ISG). Diese kann mit geeigneter Technik beseitigt werden, was zu sofortiger Besserung führt.

Eine geeignete manualmedizinische Untersuchung findet i.d. R. nicht statt. Ebenso wird keine ISG-Funktionsstörung festgestellt, was auf folgende Umstände zurückzuführen ist:

1. Das ISG wird gar nicht untersucht.

2. Die Untersuchungstechnik für das ISG ist nicht bekannt.

3. Der Patient hat keine ISG-Dysfunktion (ca. $50 \%$ der Patienten).

Neurologische oder bildgebende Untersuchungen zeigen keine pathologischen Befunde.

Es wird eher ein Psychologe als ein Osteopath bzw. Manualmediziner zu Rate gezogen, weil eine gerade erfolgte Operation an Hüfte und LWS immer noch als Kontraindikation für jegliche manuelle Behandlung gesehen wird.

In Kauf genommen werden dafür

- eine unnötig lange Beschwerdepersistenz,

- ein verzögerter Rehabilitationsfortschritt,

- ein unnötig langer Hilfebedarf und

- ein unnötig langer Analgetikabedarf.

\section{Methodik}

Die altbewährten Techniken, die überwiegend manipulativ (mit Impuls) ausgerichtet sind und/oder rotatorische Komponenten enthalten, sollen hier nicht angewandt werden. Dies liegt darin begründet, dass bei Techniken in Seitenlage und deutlicher Adduktion ein Risiko der Luxation, Materiallockerung oder -dislokation bzw. Fraktur durch beträchtliche
Krafteinleitung im Impuls sowie Scherkräfte am Oberschenkel nicht von der Hand zu weisen ist.

So wird bei der verbreiteten Technik "Panthersprung" (• Abb. 1) eine ruckartige Traktion am gestreckten Bein in Bauchlage des Patienten vorgenommen, indem dessen distaler Unterschenkel zwischen den Oberschenkeln des Therapeuten unter Gegenhalten am Sakrum eingeklemmt wird, wobei der Therapeut durch Strecken seiner Beine einen Impuls in Längsrichtung ausübt.

Diese Technik [1] weist jedoch gravierende Nachteile auf:

- Die Impulsstärke ist nicht genau dosierbar.

- Es besteht eine starke Zugbelastung für Knie und Hüfte.

- Sie ist bei einliegenden EndoproFrakturgefahr).

- Die Impulsrichtung ist nicht optimal. thesen obsolet (Lockerungs- bzw.

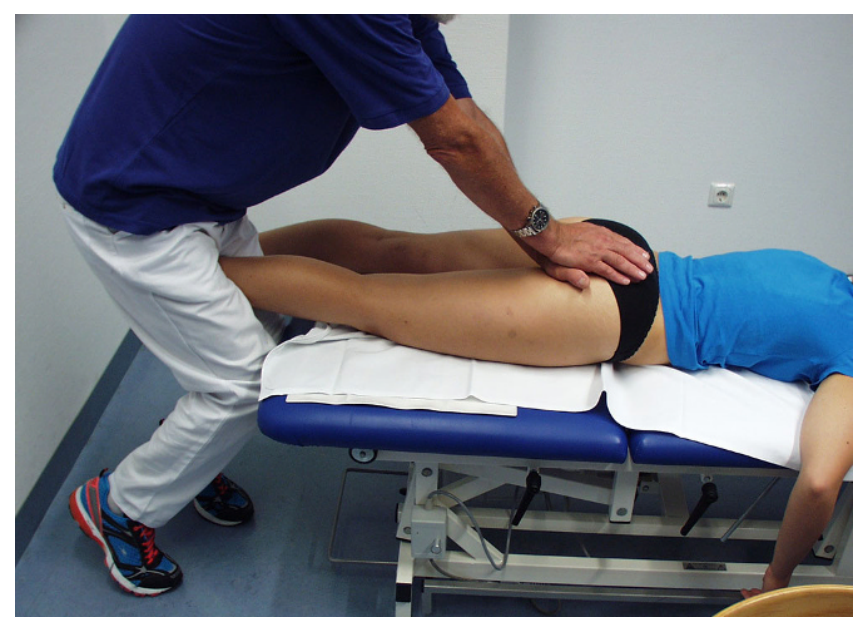

Abb. $1<$ "Panthernipulationstechnik am lliosakralgelenk sprung", eine Ma- 


\section{In der Diskussion}
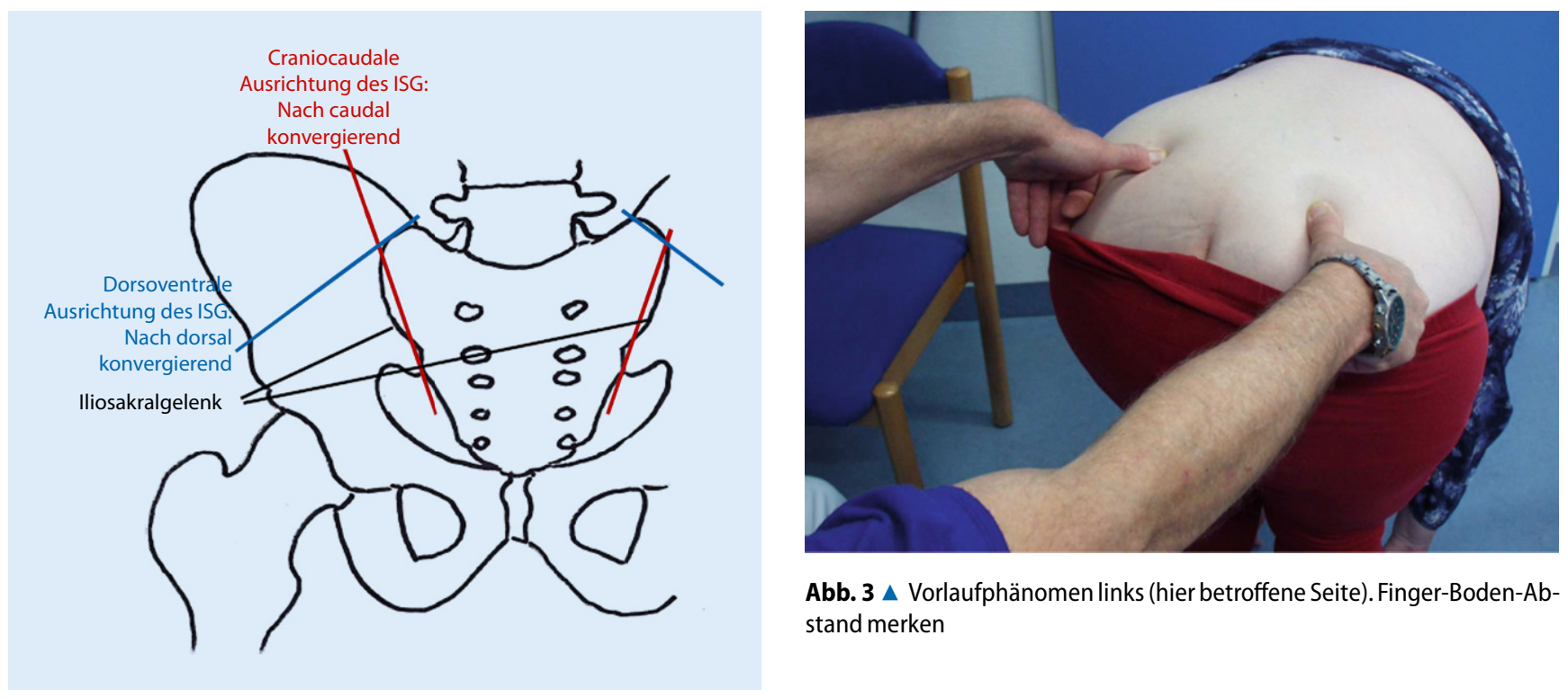

Abb. $3 \Delta$ Vorlaufphänomen links (hier betroffene Seite). Finger-Boden-Abstand merken

Abb. 2 A Räumliche Ausrichtung der lliosakralgelenke im Körper. ISG Iliosakralgelenk
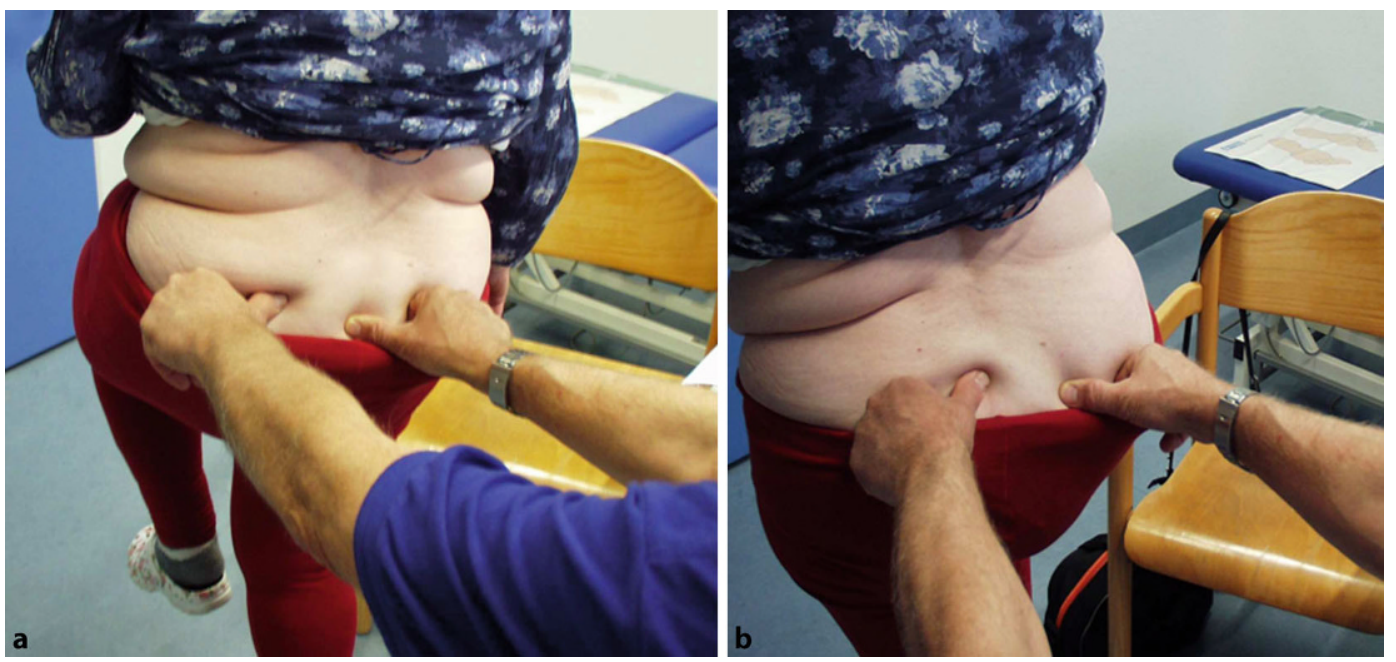

Abb. $4<$ a Spine-Test links positiv (hier betroffene Seite). b Spine-Test rechts negativ (hier nichtbetroffene Seite)

Als Alternative kommen Techniken infrage, die ohne die genannten risikobehafteten Vorgehensweisen auskommen. Eine Mobilisation - also ohne Impuls mit minimalen Bewegungsausschlägen, ggf. mit Vorbereitung durch Counterstrain-Anwendung [2,3], führt zur sicheren Deblockierung des ISG. Diese nimmt der Patient als Schmerzreduktion und verbesserte Beweglichkeit und der Untersucher als Normalisierung der klinischen Funktionstests (Vorlauftest, SpineTest, Finger-Boden-Abstand) wahr.

Die Deblockierung des ISG kann in jeder Phase der Behandlung eintreten, auch spontan schon im Ansatz einer passiven Bewegung. Oft ist das Eintreten der De- blockierung gar nicht zu bemerken. Auch ein Knackgeräusch tritt nur selten auf.

Die Bewertung der klinischen Untersuchung und die manuelle Behandlung basieren auf der räumlichen Anordnung der Iliosakralgelenke im Körper (• Abb. 2; [4]), d.h. die Ausrichtung kraniokaudal bedeutet nach kaudal konvergierend, die Ausrichtung ventrodorsal nach dorsal konvergierend.

Eine Minimalbewegung der Gelenkpartner gelingt am ehesten in der Ebene der Gelenkflächen:

- Längstraktion in Bauchlage mit leichter Adduktionsrichtung der Hüfte
- Gegennutation durch Dorsalextension der Hüfte mit leichter Adduktion

- Dorsale „Aufklappung“ durch Innenrotation der Hüfte in Bauchlage

Zur Entspannung der Glutealmuskulatur mit der Counterstrain-Technik erfolgt in Bauchlage die Positionierung für die kraniale Hälfte des ISG in Hüftadduktion und Innenrotation, für die kaudale ISGHälfte in Hüftabduktion und Außenrotation. 


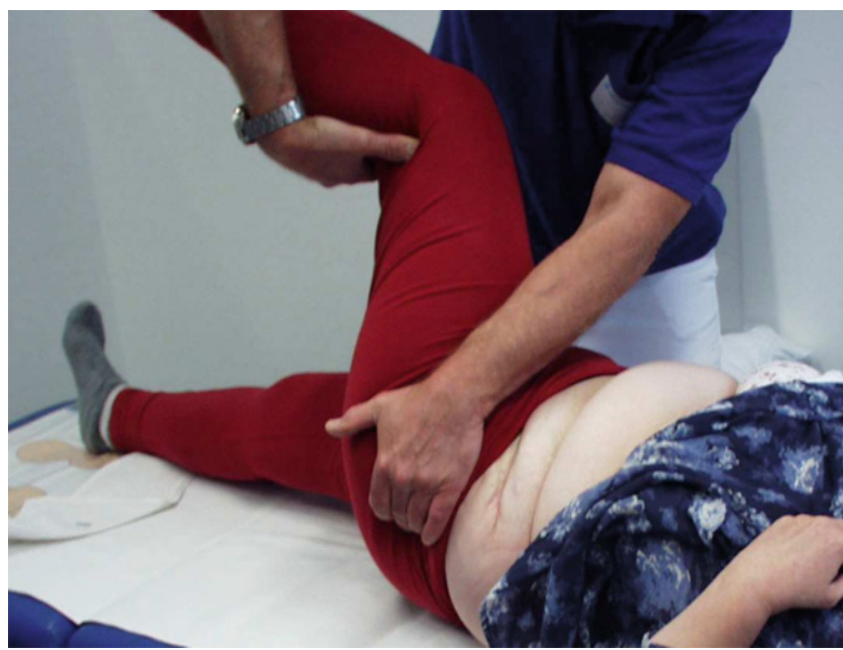

Abb. 5 In Rückenlage geschützte Adduktion und Innenrotation zur dorsalen und kaudalen „Aufklappung“ des lliosakralgelenks

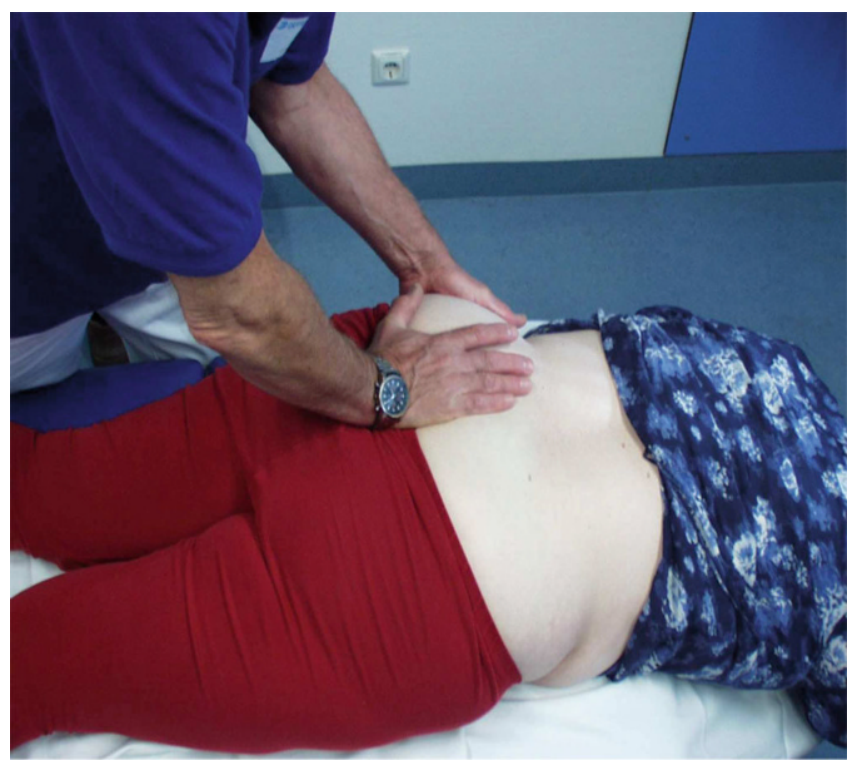

\section{Manuelle Diagnostik}

Liegt eine Dysfunktion des ISG vor, ergeben die durchgeführten Funktionstests folgende Befunde:

- Das Vorlaufphänomen auf der betroffenen Seite ist positiv (• Abb. 3).

- Der Spine-Test (bei erlaubter Vollbelastung) ist auf der betroffenen Seite positiv, auf der Gegenseite negativ (•Abb. 4).

Als sekundäres Kriterium ist der Finger-Boden-Abstand zu registrieren, da er nach erfolgter ISG-Deblockierung i. d. R. deutlich geringer ist.
Abb. $7<$ Dorsalisierung der Spina iliaca anterior superior bei fixiertem Sakrum

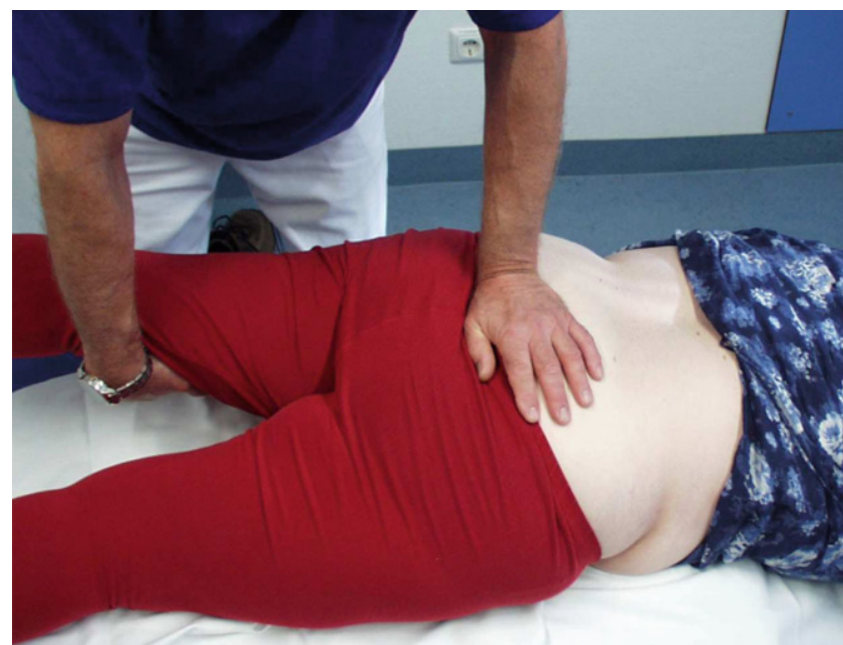

Abb. 6 A In Bauchlage bei fixiertem Sakrum wiederholte Abduktion und Adduktion bis zur Entspannung der Glutealmuskulatur (vertrauensbildende Maßnahme)

Analog wird der potenziell luxationsfördernde Effekt der Außenrotation bei der Counterstrain-Technik durch Abduktion im Hüftgelenk neutralisiert.

Auf eine therapeutische Nutzung des Patrick-Zeichens bzw. Viererzeichens [5] wird verzichtet, da die erforderliche Hüftbeweglichkeit (das betroffene Bein in Außenrotation so positioniert, dass bei gekreuzten Unterschenkeln die Beine eine „4“ bilden) ohnehin meist nicht vorliegt.

\section{Behandlung in Rückenlage}

Zunächst erfolgt die Behandlung in Rückenlage:

- Feststellung der Hüftbeweglichkeit

beidseits. Insbesondere wird mithilfe des Thomas-Handgriffs [6] ein mögliches Streckdefizit festgestellt, das in Bauchlage eine Unterfütterung des Beckens durch eine Halbrolle nötig macht, da sonst eine entspannte Lagerung nicht möglich ist.

- Orientierende neurologische Untersuchung (Sensibilität, Patellarsehnenund Achillessehnenreflex, LasègueZeichen)

- Geschützte passive Adduktion mit Innenrotation in Flexion (• Abb. 5)

- Gegebenenfalls Dorsalisierung der Beckenkämme wechselseitig oder gleichzeitig durch lokalen Druck

\section{Behandlung in Bauchlage}

Die Behandlung wird in Bauchlage fortgesetzt, die der Patient selbstständig einlung des Hüftkopfs in die Pfanne bewirkt. 


\section{In der Diskussion}
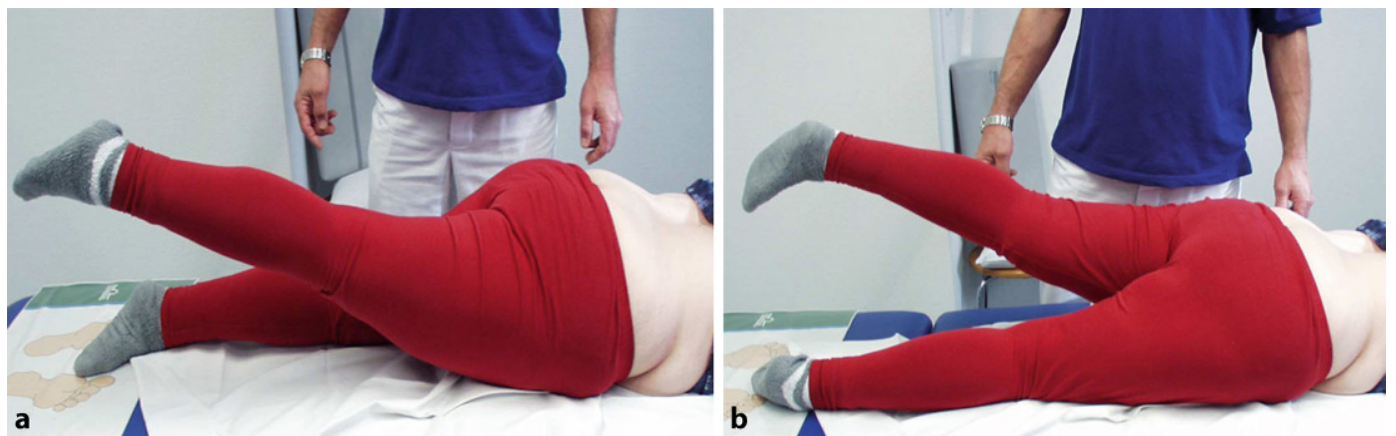

Abb. $8<$ Orientierende Erfolgskontrolle. Aktive Dorsalextension a der nichtbetroffenen Seite, $\mathbf{b}$ der betroffenen Seite
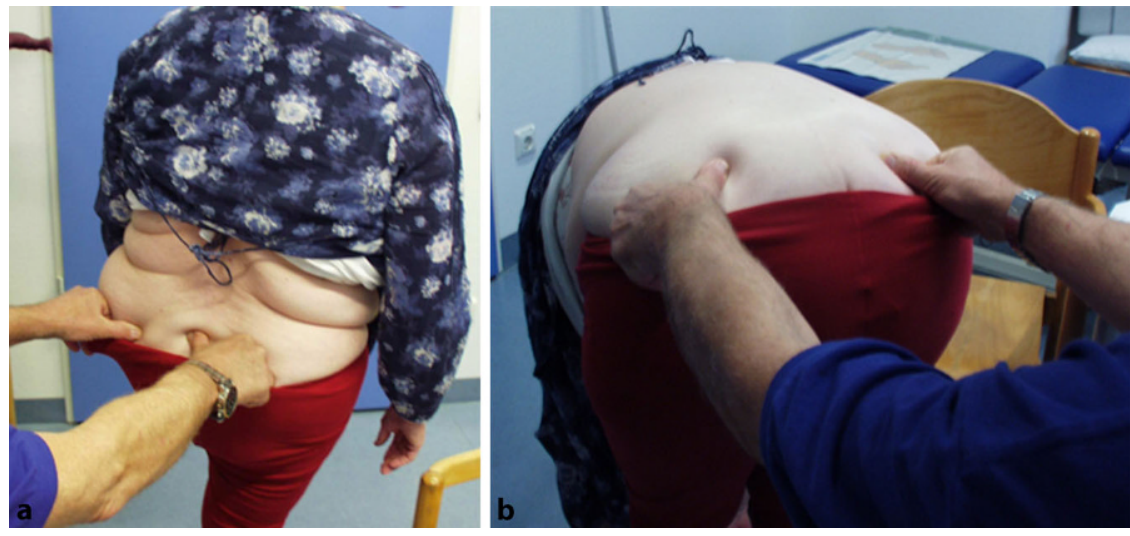

Abb. 9 ॥ Abschlussuntersuchung. a Spine-Test auf der betroffenen Seite normalisiert. b Vorlauftest seitengleich, deutlich verminderter Finger-Boden-Abstand

nehmen soll, evtl. durch Aufstehen und erneutes Hinlegen in Bauchlage. Bei Vorliegen eines Hüftstreckdefizits ohne Erreichen der Nullstellung ist die Unterfütterung des Beckens mit einer Halbrolle erforderlich.

Die Behandlung verläuft folgendermaßen:

- Horizontales wiederholtes passives

Bewegen der Beine bei fixiertem

Sakrum in Abduktion und leichter

Adduktion der Hüften (• Abb. 6),

jeweils Aufnehmen des Beins von der

Unterlage am distalen Oberschenkel

bis zur Entspannung der Gluteal-

muskulatur (vertrauensbildende

Maßnahme)

- Dorsalextension der Hüften neutral und mit leichter Adduktion in gleicher Handhaltung bei fixiertem Sakrum

- Dorsalisierung der Spina iliaca anterior superior bei fixiertem Sakrum (Nutation; - Abb. 7; [7])

- Beidseits Ventralisation des Sakrums am kaudalen ISG-Anteil (Gegennutation; [7])
- Eventuell leichte passive Traktion der Hüften in Streckstellung bei gleicher Handhaltung

- Orientierende Erfolgskontrolle: aktive Dorsalextension zunächst der nichtbetroffenen Seite als Referenzwert (๑Abb. 8a), dann der betroffenen Seite ( $\bullet \mathbf{A b b}$. 8b): Bei seitengleichen Ausschlägen Überprüfung im Stand, sonst Wiederholung der Behandlung in Bauchlage

\section{Abschlussuntersuchung}

Die Abschlussuntersuchung zeigte im vorliegenden Fall folgende Ergebnisse:

- Spine-Test auf der betroffenen Seite normalisiert (ब Abb. 9a)

- Vorlauftest seitengleich, deutlich verminderter Finger-Boden-Abstand (• Abb. 9b)

\section{Prognose}

Eine in der beschriebenen Technik beseitigte ISG-Dysfunktion kann nach einigen Tagen wieder auftreten, da die zuvor praktizierte Fehlbewegung beim Gehen auch ohne Fortbestehen des Auslösers weiter erfolgt. Dies wird zugunsten einer schonenden Behandlung bewusst in Kauf genommen. Eine nochmalige gleichartige Behandlung wird i. d. R. einen dauerhaften Erfolg bringen.

\section{Fazit}

Auch Patienten nach HTEP-Implantation oder LWS-Operation mit der eingangs angegebenen Beschwerdekonstellation sind einer geeigneten manuellen Behandlung zugänglich.

Ein Verzicht darauf aufgrund überholter Vorstellungen über manuelle Behandlung oder aus den anderen genannten Gründen wirkt sich nur zum Nachteil des Patienten aus und reduziert den erreichbaren Rehabilitationserfolg.

Eine unspektakuläre, vom erfahrenen Manualtherapeuten einfach durchzuführende manuelle Behandlung kann durchaus

- eine schmerzhafte Gangstörung beseitigen,

- Leistenschmerzen nach HTEPImplantation kurieren,

- ein sensibles Pseudo-L5/S1-Syndrom entfernen,

- eine Bewegungseinschränkung des betroffenen Hüftgelenks mindern,

- Hilfebedarf beim Strümpfeanziehen überflüssig machen und

- Schwierigkeiten beim Treppensteigen minimieren.

\section{Korrespondenzadresse}

\section{Dr. Titus Schultz}

Kirchplatz 1, 77963 Allmannsweier,

Deutschland

dr.schultz_orthopaedie@web.de 


\section{Einhaltung ethischer Richtlinien}

Interessenkonflikt. T. Schultz gibt an, dass kein Interessenkonflikt besteht.

Die abgebildete Patientin hat ihr Einverständnis zur Verwendung der Bilder gegeben.

Open Access. Dieser Artikel wird unter der Creative Commons Namensnennung 4.0 International Lizenz (http://creativecommons.org/licenses/by/4.0/deed. de) veröffentlicht, welche die Nutzung, Vervielfältigung, Bearbeitung, Verbreitung und Wiedergabe in jeglichem Medium und Format erlaubt, sofern Sie den/die ursprünglichen Autor(en) und die Quelle ordnungsgemäß nennen, einen Link zur Creative Commons Lizenz beifügen und angeben, ob Änderungen vorgenommen wurden.

\section{Literatur}

1. Bischoff HP (1988) Chirodiagnostische und chirotherapeutische Technik. perimed, Erlangen, S76

2. Greenman PE (1998) Lehrbuch der osteopathischen Medizin. Haug, Heidelberg, S 127

3. Meert GF (2003) Das Becken aus osteopathischer Sicht. Urban \& Fischer, München, Jena, S319

4. Waldeyer A (1974) Anatomie des Menschen, erster Teil, 10. Aufl. de Gruyter, Berlin, New York, S246

5. Krämer K-L, Stock M, Winter M (1992) Klinikleitfaden Orthopädie. Jungjohann, Neckarsulm, Stuttgart, $\mathrm{S} 540$

6. Debrunner HU (1987) Orthopädisches Diagnostikum, 5. Aufl. Thieme, Stuttgart, New York, S128

7. Frisch $\mathrm{H}$ (2001) Programmierte Untersuchung des Bewegungsapparates, 8. Aufl. Springer, Berlin, Heidelberg, New York, S167

\section{Aktuelle Buchempfehlungen aus dem Springer-Verlag}

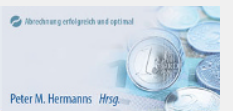

GOÄ 2019

Kommentar,

IGeL-Abrechnung
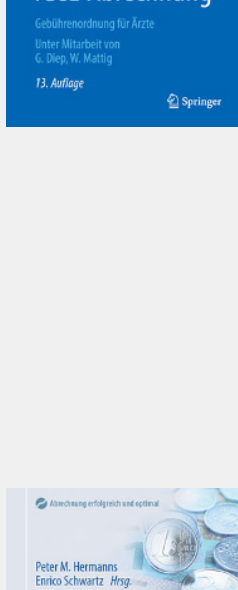

UV-GOÄ 2019

Kommenta
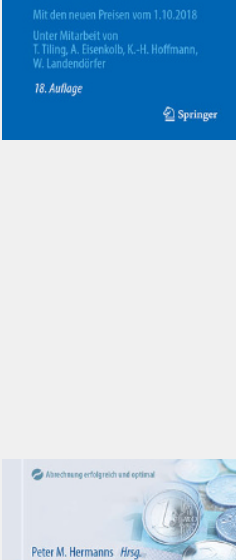

EBM 2019

Kommentar

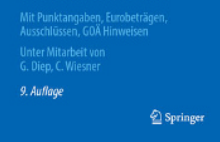

\section{GOÄ 2019 Kommentar, IGeL-Abrechnung}

\section{Gebührenordnung für Ärzte}

Hermanns, Peter M. (Hrsg.)

79,99 Euro, 2019 SPRINGER

13., vollst. üb. u. akt. Auflage

Seitenzahl XXII, 892, ISBN 978-3-662-58210-7

- Aktuelle Änderungen als pdf auf www.springermedizin.de/HermannsGOÄ

- Kommentar für Praxis und Klinik

- Mit Auslegungshinweisen der BÄK, IGeL-Hinweisen und Abrechnungsbeispielen

- Amtliche Gebührenordnung - Ausschlüsse - Analoge Bewertungen Abrechnungstipps

- Mit aktuellen Gerichtsurteilen

\section{UV-GOÄ 2019 Kommentar}

\section{Mit den neuen Preisen vom 1.10.2018}

Hermanns, Peter M., Schwartz, Enrico (Hrsg.)

69,99 Euro, 2019 SPRINGER

18., vollst. üb. u. akt. Auflage

Seitenzahl XXIII, 743, ISBN 978-3-662-58268-8

- Änderungen zum Vertrag Ärzte/UV-Träger ab 1.1.2019 als pdf auf www.springermedizin.de/HermannsUV-GOÄ

- Zuständigkeit der UV-Träger auf einen Blick

- Alle praxisrelevanten Kommentierungen, inklusive aktuelle Gerichtsurteile

- Mit Abrechnung bei Arbeitsunfällen und Berufskrankheiten

- Korrekt, verlässlich, vollständig

\section{EBM 2019 Kommenta}

Mit Punktangaben, Eurobeträgen, Ausschlüssen,

\section{GOÄ Hinweisen}

Hermanns, Peter M. (Hrsg.)

79,99 Euro, 2019 SPRINGER

9., vollst. üb. u. akt. Aufl.

SeitenzahI XXI, 903, ISBN 978-3-662-58270-1

- Aktuelle Änderungen als pdf auf www.springermedizin.de/HermannsEBM

- Vollständige Leistungslegenden mit Bewertungen

- Ausschlüsse

- Wer darf was wann im EBM abrechnen

- Praktische Abrechnungstipps und -beispiele mit Auslegungshinweisen, Beschlüssen, Richtlinien, Texten, u.a. von KBV und regionalen KVen, G-BA, Sozialgesetzbuch (SGB) 